\title{
Meeting report: Pre-exposure Human Immunodeficiency Virus Prophylaxis in the EU/EEA: Challenges and Opportunities, Stockholm April 2016
}

T Noori ${ }^{1}$, A Pharris ${ }^{1}$

1. European Centre for Disease Prevention and Control (ECDC), Stockholm, Sweden

Correspondence: Teymur Noori (teymur.noori@ecdc.europa.eu)

The ECDC held an expert meeting in Stockholm on 27-28 April 2016 to discuss practical considerations for pre-exposure prophylaxis (PrEP) implementation in Europe. The meeting focused on four key areas: (i) eligibility criteria for PrEP in Europe; (ii) appropriate models of service delivery; (iii) cost-effectiveness of PrEP, and (iv) routine monitoring of people on PrEP.

PrEP is the regular use of an antiretroviral medication by people who are uninfected to prevent the acquisition of HIV infection. Currently Emtricitabine/Tenofovir Disoproxil Fumarate (TDF/FTC) or tenofovir alone is used. Since 2010, the efficacy of oral PrEP has been shown in four randomised controlled trials [1-4]. In 2015, the World Health Organization (WHO) recommended that PrEP should be offered as an additional prevention option for people at substantial risk of HIV infection as part of combination prevention approaches [5].

In the European Union/European Economic Area (EU/ EEA), men who have sex with men (MSM) are disproportionately affected by human immunodeficiency virus (HIV) and other sexually transmitted infections (STI) $[6,7]$. Consequently, strengthening efforts to reduce the incidence of HIV and STI among MSM is a priority for the European Centre for Disease Prevention and Control (ECDC), which recently published comprehensive guidance on HIV and STI prevention among MSM [8] and an opinion encouraging countries to consider integrating PrEP into their existing HIV prevention packages for those most at-risk of HIV infection, starting with MSM.

\section{Eligibility criteria for pre-exposure} prophylaxis in Europe

Elske Hoornenborg from the AMPrEP project in Amsterdam, the Netherlands, provided an overview of eligibility criteria for PrEP. Review of PrEP studies, demonstration projects and existing guidelines show that eligibility criteria are very similar. WHO guidelines recommend PrEP for population groups with HIV incidence >3\%; United States (US) Centers for Disease Control and Prevention guidelines recommend PrEP for MSM at substantial risk of HIV, and European AIDS Clinical Society (EACS) guidelines recommend PrEP for MSM or transgender people with inconsistent condom use with casual partners or an HIV positive partner not on treatment, with recent STI or use of post-exposure prophylaxis (PEP) $[5,9,10]$.

Key issues emerging from the presentation and following discussion include:

- Eligibility criteria may need to be adapted to reflect the epidemiological context, since population groups at high risk of HIV differ between countries in Europe. MSM at high risk for HIV acquisition are a key group for which PrEP is being considered in many EU/EEA countries.

- The need (i.e. those at high risk of HIV) and demand (i.e. those coming forward for PrEP or accepting if offered) of PrEP should be considered separately when formulating eligibility criteria.

- Eligibility criteria should ensure that PrEP use maximises public health benefit and cost- effectiveness.

- Some country representatives expressed concerns about people who do not meet eligibility criteria but are still obtaining PrEP. However, the evidence to-date suggests that most MSM seeking PrEP selfselect, i.e. they are at high risk of HIV.

\section{Appropriate models of service delivery}

Sheena McCormack, from University College London (UCL), United Kingdom (UK), presented an overview of options for delivering PrEP, including delivery in clinicbased services, community-based services, by HIV 
specialists, primary care physicians, peers and online. She pointed out that whichever model is chosen, consideration must be given to suitable systems for purchasing drugs, additional resource requirements and how best to integrate PrEP into existing services. Integrating PrEP should be relatively straightforward for countries with services offering HIV and STI diagnosis and treatment and PEP, as PrEP is relatively simple to prescribe as there are limited drug choices and few side effects or drug interactions.

Key issues emerging from the presentation and following discussion include:

- Feasible options will depend on the country context and the way in which the health system is organised. In some countries, primary care physicians provide HIV and STI treatment and care and could deliver PrEP, but, in others, HIV care and follow up is provided by HIV or infectious diseases specialists.

- Given differences in country contexts, it is not feasible to make Europe-wide recommendations. Each country will need to consider where HIV/STI testing and treatment are best delivered. However, European guidance on general principles and minimum standards, e.g. for safe prescribing, quality of care and monitoring, and maximising the benefits of PrEP as a prevention tool, would be helpful.

- Encouraging people who are at risk but who are HIV negative to engage with health services is critical, and MSM-friendly services can facilitate this.

- Community-based services should have appropriate referral links and pathways in place to ensure that people on PrEP receive follow-up care and routine monitoring. Specific concerns about online delivery of PrEP include how to promote adherence and provide follow-up care, as well as how to ensure that people are purchasing genuine drugs and reduce the risks associated with stock outs of drugs.

\section{Cost and cost-effectiveness of PrEP}

Valentina Cambiano (UCL) and Nigel Field (UCL and Public Health England, UK) presented work on the costeffectiveness of PrEP among MSM in the UK, using two different models, and the work by Brooke Nichols and colleagues (Erasmus Medical Center, Rotterdam) in the Netherlands.

Available evidence suggests that significant reductions in drug prices will be needed for PrEP to be considered cost-effective (now) if the time horizon under consideration is only short-medium term. However, each infection averted now is averting health service antiretroviral therapy costs for many decades to come and so it is appropriate to consider a long-term time scale (e.g. 80 years). Based on the modelling conducted by Cambiano and colleagues and by Nichols and colleagues, PrEP is likely to prove to be cost effective, although in the Netherlands only if PrEP is taken on demand considering a long time horizon. Presenters pointed out that making the public health case for an intervention such as PrEP, which has a substantial short-term budget impact but potential for substantial longer-term savings in cost and public health benefit, is challenging.

Key issues emerging from the presentation and following discussion include:

- Demonstrating the impact of PrEP on new HIV infections outside of clinical trials will be critical. Positive results from France, where PrEP is currently implemented, and from demonstration projects showing a reduction in new infections will be important evidence to aid decision makers considering PrEP.

- As individual countries might need to conduct their own cost-effectiveness studies, some guidance to standardise these cost-effectiveness studies would be useful. Some participants in the meeting were doubtful whether the cost-effectiveness arguments would be of value in convincing policymakers, as decisions are more strongly influenced by the shortterm budget impact.

- The cost of the drugs is the key barrier to free provision of PrEP by public health services. Costs are expected to drop once generic drugs become available in Europe.

\section{Monitoring of people on PrEP}

The key points related to routine clinical and public health monitoring of people on PrEP such as adherence, drug resistance and regular STI screening were covered in three presentations.

Pep Coll (Barcelona Checkpoint, Spain) provided an overview of the evidence about adherence to PrEP. Studies have shown that PrEP is efficacious if it is taken as prescribed (in the range of $90 \%$ ). Ensuring adherence to the dosing regimen is crucial whether PrEP is taken daily or on demand. The barriers to adherence include stigma, lack of community acceptance of PrEP, the need to conceal PrEP use, chemsex, mental health problems, social factors, and mobility.

Robert Grant (University of California, San Francisco, US) discussed the issue of drug resistance in the context of PrEP. Concerns have been raised that generalised or inappropriate PrEP use could result in the development and transmission of drug-resistant strains of HIV. Drug resistance during PrEP use and PrEP trials has been low. A systematic review of drug resistance in PrEP trials found that there were five cases of incident drug resistance in 9,222 people in the active PrEP arms, i.e. the overall risk of resistance was $0.5 \%$. The risk of drug resistance is higher in people with acute HIV infection when they start PrEP, i.e. in the window period, but is low in those who seroconvert while taking PrEP. There 
is a case report of oral FTC/TDF PrEP failure to prevent HIV infection despite good adherence. This was a very particular case involving the acquisition of an extensively resistant virus mutated strain. One strategy to mitigate the risk of drug resistance could be the use of more sensitive assays to detect acute HIV infection in the window period.

In her second talk, Sheena McCormack discussed the impact on other STIs following the introduction of PrEP. Both overall European Union/European Economic Area (EU/EEA) and UK data show that bacterial STIs were increasing among MSM before PrEP, particularly among high risk MSM. Data from the UK PROUD study, which was conducted among HIV-negative MSM with a high burden of self-reported STI, show that there was no difference in the proportion with an STI between those on PrEP and those not on PrEP after 12 months, with both groups followed up for HIV and STI every 3 months. The incidence of STIs among MSM is increasing and is likely to continue to increase in Europe with or without PrEP. PrEP can contribute positively to STI control by increasing regular asymptomatic screening, prompt treatment and partner notification, at the same time as providing support to MSM who want to reduce risk behaviour.

Key issues emerging from the presentations and following discussions include:

- Lack of access to PrEP through health services will contribute to adherence problems, because if users are purchasing PrEP online they might not receive quality products, or find it hard to continue to pay or the supplies will not be reliable.

- The rise in practicing condomless sex resulting in greater exposure to STIs by those on PrEP is of concern to some stakeholders. In particular there are concerns that widespread PrEP use could lead to an increase in the incidence of MDR gonorrhoea, although this has not been seen in the US or in France so far. In France, rates of gonorrhoea among PrEP users have actually dropped even though rates of testing have increased in this risk group. In the UK, data from the PROUD study indicates that there is still a good level of condom use in this risk group.

- Clear evidence and messages to various stakeholders (policymakers, public health experts, clinicians, community representatives, etc.) about PrEP and STIs (e.g. that STI rates are already high in those MSM who would benefit most from PrEP, or that rates of STI are increasing with or without PrEP) will be critical. However, an additional increase in STIs is still likely (as was the case historically with similar major developments such as the introduction of oral contraceptives) and the health services need to plan for this eventuality.
- PrEP should be provided as part of a comprehensive package which will also allow for earlier diagnosis and linkage to care of STIs and for other interventions that may reduce the incidence of STIs.

- Surveillance systems should be adapted in order to monitor the use of PrEP, including use outside public health systems, and PrEP failures to ensure suitable measures are carried out to maximise the effectiveness of this prevention strategy.

\section{Conclusions}

PrEP should not be considered in isolation but as an additional option for people at substantial risk of HIV infection as one element of a combination prevention approach. There may be several models of service provision that may deliver PrEP effectively to those population groups at highest risk of HIV and the final choice will be determined by the specificities and organisation of countries' health services. The current cost of PrEP remains the main obstacle for implementation in the European setting. The second main obstacle is the potential impact of PrEP on risk behaviour by an already high risk population. However, a well-planned PrEP service will make good use of the need for PrEP users to attend regular check-ups ensuring prompt diagnosis, treatment and the offer of partner notification, while providing specific support to MSM who want to reduce their risk behaviour. Meeting participants identified a number of priority activities that that could be considered to support policy and implementation of PrEP in the EU/EEA, including: updating the current ECDC evidence-based guidance on HIV and STI prevention among MSM to include the new evidence on PrEP implementation; developing a model/tool to support comparable national cost-effectiveness studies; working with Member States to identify minimum standards and principles for service delivery; exploring the possibility of using national surveillance data to estimate the number of people in need of PrEP; identify standard indicators to monitor PrEP and explore the potential to use European HIV cohorts to monitor PrEP use and impact.

\section{Acknowledgements}

Andrew Amato-Gauci, Kathy Attawell, Manuel Battegay, Josip Begovac, Torsten Berglund, Anders Blaxhult, Viviane Bremer, Gus Cairns, Valentina Cambiano, Jordi Casabona, Olivia Castillo Soria, Pep Coll, Susan Cowan, Caroline Daamen, Silke David, Antonio Diniz, Nigel Field, Robert Grant, Neta Harel, Elske Hoornenborg, Kristina Ingmarsdotter Persson, Cary James, Marie Laga, Lennie Lindberg, Fiona Lyons, Bruno Maia, Anna Marzec-Bogustawska, Sheena McCormack, Michael Meulbroek, Jean-Michel Molina, Arild Johan Myrberg, Otilia Mårdh, Brooke Nichols, Georgios Nikolopoulos, Velina Pendolovska, Ines Perea, Francois Pichon, Ferran Pujol, Daniela Rojas Castro, Gianfranco Spiteri, Anders Sönnerborg, Judit Takacs, Lara Tavoschi and Marti Vall.

Conflict of interest 
TN wrote the first draft of the manuscript. AP critically reviewed the paper and gave input to the content, which was incorporated in the report. Both authors read and approved the final manuscript.

\section{References}

1. Grant RM, Lama JR, Anderson PL, McMahan V, Liu AY, Vargas $L$, et al. , iPrEx Study Team. Preexposure chemoprophylaxis for HIV prevention in men who have sex with men.N Engl J Med. 2010;363(27):2587-99. DOI: 10.1056/NEJMoa1011205 PMID: 21091279

2. Baeten JM, Donnell D, Ndase P, Mugo NR, Campbell JD, Wangisi l, et al. Partners PrEP Study Team. Antiretroviral prophylaxis for HIV prevention in heterosexual men and women.N Engl J Med. 2012;367(5):399-410. DOI: 10.1056/NEJMoa1108524 PMID: 22784037

3. McCormack S, Dunn DT, Desai M, Dolling DI, Gafos M, Gilson $\mathrm{R}$, et al. Pre-exposure prophylaxis to prevent the acquisition of HIV-1 infection (PROUD): effectiveness results from the pilot phase of a pragmatic open-label randomised trial. Lancet. 2016;387(10013):53-60. DOI: 10.1016/S0140-6736(15)00056-2 PMID: 26364263

4. Molina JM, Capitant C, Spire B, Pialoux G, Cotte L, Charreau I, et al., ANRS IPERGAY Study Group. On-Demand Preexposure Prophylaxis in Men at High Risk for HIV-1 Infection.N Engl J Med. 2015;373(23):2237-46. DOI: 10.1056/NEJMoa1506273 PMID: 26624850

5. World Health Organization (WHO). Guideline on When to Start Antiretroviral Therapy and on Pre-Exposure Prophylaxis for HIV. Geneva: WHO; 2015.

6. European Centre for Disease Prevention and Control (ECDC), WHO Regional Office for Europe (WHO). HIV/AIDS Surveillance in Europe 2014. Stockholm: ECDC/WHO; 2015.

7. European Centre for Disease Prevention and Control (ECDC). Sexually transmitted infections in Europe 2013. Stockholm: ECDC; 2013.

8. European Centre for Disease Prevention and Control (ECDC). HIV and STI prevention among men who have sex with men. Stockholm: ECDC; 2015.

9. United States Centers for Disease Control and Prevention (CDC). Pre-exposure prophylaxis for the prevention of HIV infection in the United States, 2014. Atlanta: CDC; 2014.

10. European AIDS Clinical Society (EACS). EACS Guidelines, Version 8.0. Brussels: EACS, 2015.

\section{License and copyright}

This is an open-access article distributed under the terms of the Creative Commons Attribution (CC BY 4.0) Licence. You may share and adapt the material, but must give appropriate credit to the source, provide a link to the licence, and indicate if changes were made.

This article is copyright of the European Centre for Disease Prevention and Control, 2016. 